\title{
Do Environmental Factors (Practice and Feedback) Moderate the Effect of the Val66met BDNF Polymorphism on Motor Learning?
}

\author{
Abolfazl Shayan Nooshabadi ${ }^{1}$, Alireza Saberi Kakhki ${ }^{*}$, \\ Mehdi Sohrabi ${ }^{1}$ and Mohammad Ali Dowlati ${ }^{2}$ \\ ${ }^{1}$ Department of Motor Behavior, Faculty of Sport Sciences, \\ Ferdowsi University of Mashhad, Mashhad, Iran. \\ ${ }^{2}$ Research Center for Biochemistry and Nutrition in Metabolic Diseases, \\ Kashan University of Medical Sciences and Health Services, Kashan, Iran.
}

http://dx.doi.org/10.13005/bbra/2130

(Received: 17 March 2016; accepted: 20 April 2016)

\begin{abstract}
Motor learning might be affected by environmental factors as well as some genetic factors. The aim of the present study is to examine the effect of val66met BDNF polymorphism on motor learning and to examine the possibility of moderating this effect using environmental factors. One hundred students from University of Kashan, Iran participated in the study. After extraction of Genomic DNA, implementation of polymerase chain reaction (PCR), analyzing PCR by 1.5 percent Electrophoresis Gel, and in the end sequencing by ABI PRISM 7000 Sequencing Analyzer, 46 participants were identified without val66met polymorphism while 54 participants were affected by the polymorphism (met-carrier). Twenty-four participants without val66met polymorphism and Twenty-four participants of met-carriers were randomly selected and divided into four groups of twelve. Participants of each group practiced backhand baseball pitch for six sessions and after 48 hours did the first retention test. Subsequently, participants in all four groups continued practicing in three additional sessions with specific design for each group and afterwards did the second retention test. Results demonstrate the fact that participants without the polymorphism exceeded met-carriers in learning of the task. Whereas the weakness of met-carriers did not disappear by additional practice, the practice was useful when associated with self-controlled feedback. The research shows val66met polymorphism may exert an influence over the learning of motor skills. However, the effect may be moderated by changing the condition of practice for people affected by the polymorphism in a way that engages them to cognitive processes.
\end{abstract}

Key words: BDNF, Val66met Polymorphism, Motor Learning, Feedback Control, Met-carriers

Many environmental factors may affect learning of a motor skill, most notably the amount of practice and feedback ${ }^{1}$. The amount of practice is the most important factor while the manner in which the feedback is provided seems to be significant as well. For instance, it has been demonstrated that providing immediate feedback will be less effective than delayed feedback ${ }^{2}$. Some

\footnotetext{
* To whom all correspondence should be addressed. Tel: 985138805000;

E-mail: askakhki@um.ac.ir
}

studies, however, have shown some physiologic variables may have notable effect on motor learning by specific mechanisms ${ }^{3}$. One of these variables is brain-derived neurotrophic factors (BDNF). BDNF is one of the most abundant neurotrophic factors in various structures of the adult brain associated with synaptic plasticity, learning and memory ${ }^{4}$. BDNF's influence on synaptic plasticity, with a known role in the induction and maintenance of memory and learning, is due to its capacity to regulate vital synaptic functions such as synaptic transmission, neuronal survival, differentiation, and development ${ }^{5}$. 
Since it is impossible to investigate a human brain directly, the effect of BDNF in humans has in large part been revealed through studying individuals with a single nucleotide polymorphism (SNP) of the BDNF gene, which results in a valineto-methionine substitution at codon 66 (val66met) ${ }^{5}$. The advent or absence of SNP genetically divides people into three categories: people with two valine at codon 66 (val/val), people with a valine and a methionine (val/met), and people with two methionine (met/met). People of the first category have not been affected by the polymorphism and are referred to as people without val66met polymorphism. Members of the two latter categories have been affected by the polymorphism and are called met-carriers. While the polymorphism does not alter the mature BDNF structure, it impairs its trafficking. This defect have been associated with reduced activity-dependent BDNF release ${ }^{4}$, decreased hippocampal volume ${ }^{7}$, and impaired episodic memory ${ }^{4}$, between metcarriers.

Due to the vital role of BDNF in facilitation of the processes related to motor learning ${ }^{[5]}$ and in formation of long term potentiation (LTP), known as long term synaptic plasticity or motor memory consolidation $^{8}$, it is tempting to hypothesize that the polymorphism might affect motor learning. McHughen et al., (2010) investigated the motion of the index finger performing a motor task using fMRI and concluded met-carriers have lower activation volume than others in several brain areas $^{9}$. The excellence of people without the polymorphism has also been shown by Joundi et al., (2012), in learning of visuomotor adaptation task $^{5}$. Moreover, in another study using BOLD fMRI, met-carriers exhibited relatively diminished hippocampal engagement in comparison with people without val66met polymorphism during both encoding and retrieval processes ${ }^{10}$. However, Freundlieb et al., (2012) found no difference between two groups in motor learning and vocabulary learning ${ }^{6}$. Furthermore, in a different research design, after one day of practice of the marble navigation task McHughen et al., (2011) showed people without the polymorphism were better than met-carriers, while if the practice was continued for five days, the difference disappeared. This formula was repeated in the short-term cortical motor map plasticity evaluated by transcranial magnetic stimulus (TMS) ${ }^{11}$.

Therefore, primarily due to lack of studies on motor learning, simplicity of selected task at the studies, and probable difference in prevalence and behavioral effectiveness of BDNF genotype in various ethnic groups ${ }^{12-14}$, researchers sought to answer the question, "Does the existence of val66met polymorphism in an Iranian society influence motor learning or not?". Secondarily, due to the importance of environmental variables in motor learning, researchers sought to answer the question of whether or not influence of the genetic factor on motor learning is moderated by induction of the most important environmental variables.

\section{MATERIALS AND METHODS}

\section{Subjects}

One hundred Iranian students from university of Kashan, Iran (mean age: $21.60 \pm 2.20$ years; age range: $19-25$ years) participated in the study. The research process was described and the written informed consent was taken. The experiments reported in this study were performed in accordance with the ethical standards of the Helsinki Declaration. None of the participants had a history of serious medical, neurological or psychiatric illness, behavioral and motor problems or use of illegal, neuroactive or recreational drugs ( $>15$ cigarettes/day, $>6$ cups of coffee/day, $>50 \mathrm{~g}$ of alcohol/day) 6 . For a closer look, the groups were homogenized by participant sport history and Body Mass Index (BMI). Moreover, considering the possible effect of sex hormones on regulating the expression of BDNF ${ }^{15,16}$, just men and bachelors were selected.

\section{BDNF genotyping}

Genomic DNA was extracted from whole blood by the DNA extraction kit (Gene All Company). The Val66Met SNP in the BDNF gene was typed by polymerase chain reaction (PCR) amplification. The materials used to do PCR were as follows; $1 \mu \mathrm{l}$ of template DNA, $1 \mu \mathrm{l}$ forward primer, $1 \mu \mathrm{l}$ reverse primer, $12.5 \mu \mathrm{l}$ Master Mix 2x (The red Ampliqun of Gene All Company), and $9.5 \mu$ distilled water. Primer sequences were forward: 5ACTCTGGAGAGCGTGAAT-3 and reverse: 5ATACTGTCACACACGCTG-3. PCR started with an initial denaturation at $95^{\circ} \mathrm{C}$ for $12 \mathrm{~min}$, followed by denaturation at $95^{\circ} \mathrm{C}$ for $30 \mathrm{~s}$, annealing at $60^{\circ} \mathrm{C}$ 
for $45 \mathrm{~s}$, and extension at $72^{\circ} \mathrm{C}$ for $60 \mathrm{~s}$ for 30 cycles, and it ended with a final extension at $72^{\circ} \mathrm{C}$ for 4 min. The process of PCR was done by American Lab Net Thermal Cycler machine. PCR products were confirmed by $1.5 \%$ Electrophoresis Gel and then were sequenced by ABI PRISM 7000 Sequencing Analyzer. Checking of analysis sorted participants to three genetic categories: val/val (46 people), val/met (40 people), and met/met (14 people). The genotype's distribution of Val66Met polymorphism observed in both val/val and val/ met genotype satisfied the Hardy-Weinberg Equilibrium and were comparable with that previously observed in previous studies ${ }^{17-19}$. The experiment leader in this study was blinded for the genotype of the participants.

\section{Backhand baseball pitch task}

The task was to throw a ball to the middle of a square $1.7 \times 1.7-\mathrm{m}$ vertical target placed $5.0 \mathrm{~m}$ away as described in Horn et al., (2007). ${ }^{[20]}$

\section{Design and procedure}

People carrying two methionine were eliminated and then 24 participants without val66met polymorphism and 24 participants of metcarriers (val/met) were randomly selected and divided into four groups of twelve. Before the pretest, participants were told that the task was to throw the ball at maximum speed, ensuring that the ball hit the target. After six pre-test trials, participants continued to practice. Before the start of practice, participants observed five demonstrations of a model. In addition to the five consecutive demonstrations of the model, participants observed one demonstration after each of the first five acquisition trials.

In the first phase of practice, participants in the four groups similarly performed three of 12 trials on 6 consecutive sessions in 6 days (The number of trials and sessions had been set by pilot) with visual internal feedback (knowledge of result (KR)). The error score of the third 12 trials of each session was recorded. 48 hours after the last session of practice, retention test was done (12 trials). Then, at the second phase, participants in the four groups continued practice in additional three days with specific design for each group. One of the groups without val66met polymorphism (group "A") did not do any practice, another group without val66met polymorphism (group "B") and one of the met-carriers groups (group "C") continued practicing similar to the first phase of practice, and another group of the met-carriers (group “D”) continued practicing similarly but with self-controlled KR. In the last case, while the ball was released from the hand, the lights of the practice room were died out by electric eye which was on the track of the hand. Then, only when participants ask for KR, the KR was given to them. After 48 hours of the last session of practice, the second retention test was done exactly like the first one. The distance to the target was considered as the error score. It should be noted that due to some problems to complete training by three participants, their data were eliminated.

\section{Statistical analysis}

We used a Kolmogorov Smirnov Test to assess the normality of distribution of data. Then, a repeated measures analysis of variance (ANOVA) with factor of "practice session" was used to test changes in performance of participant at the acquisition phases. One-way ANOVA was used to test differences between groups in the pre-test and the retention tests. For all statistical analyses $\mathrm{P} \leq 0.05$ were considered to indicate statistical significance.

\section{RESULTS}

\section{Demographic and descriptive statistics have been summarized in table 1}

One-way ANOVA showed a significant difference between the score of groups just at the first retention test $(\mathrm{F}=3.270, \mathrm{p}=0.033)$ (table 2). Post hoc analysis showed that the difference is significant just between "A" and "D" groups (table 3).

Repeated measures ANOVA for the first acquisition showed significant effect of "practice session” ( $F=15.809$, pÂ0.001), no significant effect of group ( $\mathrm{F}=2.306, \mathrm{p}=0.095)$, and also no significant interaction effect between group and "practice session" ( $F=1.006, p=0.392)$. In the second acquisition just effect of group is significant ( $F=10.677, \mathrm{p}<0.001$ ) (table 4).

\section{DISCUSSION}

The purpose of the present study is firstly to examine the effect of BDNF genotype on learning of complex motor skill and secondly to examine the 
Table 1. Demographic Statistic (abundance) and Descriptive Data (M $\pm \mathrm{SD})$

\begin{tabular}{|c|c|c|c|c|}
\hline & Group “A” & Group “B” & Group “C” & Group “D” \\
\hline Number & 12 & 11 & 11 & 11 \\
\hline Age (year) & $22.20 \pm 2.30$ & $21.11 \pm 2.62$ & $20.78 \pm 1.99$ & $22.22 \pm 2.68$ \\
\hline Height (m) & $1.77 \pm 0.06$ & $1.78 \pm 0.02$ & $1.77 \pm 0.07$ & $1.78 \pm 0.06$ \\
\hline Weight (kg) & $73.70 \pm 12.86$ & $71.22 \pm 11.14$ & $69.33 \pm 11.03$ & $69.22 \pm 10.46$ \\
\hline \multicolumn{5}{|l|}{ Sport history } \\
\hline City or school & 8 (person) & 8 & 8 & 8 \\
\hline provincial & 1 & 1 & 1 & 1 \\
\hline none & 3 & 2 & 2 & 2 \\
\hline \multicolumn{5}{|l|}{ BMI } \\
\hline $\mathrm{BMI} \leq 20$ & 3 (person) & 3 & 3 & 3 \\
\hline $20<\mathrm{BMI}<25$ & 7 & 7 & 7 & 7 \\
\hline $25<\mathrm{BMI}<30$ & 2 & 1 & 1 & 1 \\
\hline Error Score of Pre-test (CM) & $48.91 \pm 7.01$ & $48.42 \pm 11.82$ & $50.36 \pm 11.26$ & $51.54 \pm 12.77$ \\
\hline Error Score of 1st Session & $29.54 \pm 4.30$ & $28.75 \pm 7.91$ & $30.41 \pm 9.73$ & $32.33 \pm 10.60$ \\
\hline Error Score of 2nd Session & $21.34 \pm 3.36$ & $25.90 \pm 4.78$ & $28.67 \pm 10.30$ & $26.70 \pm 6.12$ \\
\hline Error Score of 3rd Session & $19.36 \pm 8.10$ & $26.97 \pm 9.43$ & $25.66 \pm 7.91$ & $25.18 \pm 7.95$ \\
\hline Error Score of 4th Session & $16.43 \pm 3.97$ & $26.76 \pm 9.62$ & $24.33 \pm 8.25$ & $22.92 \pm 6.62$ \\
\hline Error Score of 5th Session & $16.51 \pm 4.03$ & $21.92 \pm 6.94$ & $20.38 \pm 6.41$ & $24.81 \pm 4.58$ \\
\hline Error Score of 6th Session & $16.09 \pm 4.06$ & $22.23 \pm 7.01$ & $22.05 \pm 6.11$ & $23.09 \pm 4.51$ \\
\hline Error Score of 1st Retention & $16.54 \pm 5.36$ & $22.52 \pm 8.01$ & $22.55 \pm 7.32$ & $25.64 \pm 5.96$ \\
\hline Error Score of 1st additional Session & - & $21.71 \pm 8.49$ & $24.62 \pm 9.79$ & $35.80 \pm 6.72$ \\
\hline Error Score of 2nd additional Session & - & $17.51 \pm 4.88$ & $23.07 \pm 6.56$ & $35.52 \pm 13.22$ \\
\hline Error Score of 3rd additional Session & - & $19.05 \pm 7.77$ & $21.15 \pm 6.01$ & $36.65 \pm 21.85$ \\
\hline Error Score of 2nd Retention & $15.56 \pm 5.36$ & $19.53 \pm 8.54$ & $21.36 \pm 6.10$ & $22.88 \pm 5.07$ \\
\hline
\end{tabular}

Table 2. One way ANOVA for the error score of groups at the pre-test and the retention tests

\begin{tabular}{lcccc}
\hline & df & Mean Square & F & Sig. \\
\hline Pre-test & 3 & 22.347 & 0.189 & 0.903 \\
$1^{\text {st }}$ retention test & 3 & $168.525^{*}$ & 3.736 & 0.018 \\
$2^{\text {nd }}$ retention test & 3 & 115.871 & 2.650 & 0.061 \\
\hline
\end{tabular}

*. The mean difference is significant at the 0.05 level

Table 3. Bonferroni test for difference between error score of groups at the 1st retention test

\begin{tabular}{lccccc}
\hline & Group 1 & Group 2 & Mean Difference (1-2) & Std. Error & Sig. \\
\hline $1^{\text {st } \text { retention test }}$ & A & B & -5.98 & 2.803 & 0.234 \\
& & C & -6.01 & 2.803 & 0.227 \\
& B & D & $-9.10^{*}$ & 2.803 & 0.014 \\
& C & D & -0.03 & 2.863 & 0.999 \\
& C & D & -3.12 & 2.863 & 0.999 \\
& & & & 2.863 & 0.999
\end{tabular}

\footnotetext{
*. The mean difference is significant at the 0.05 level
} 
Table 4. Repeated measures ANOVA for the acquisition phase

\begin{tabular}{llccccc}
\hline & source & df & Mean Square & F & Sig. & Partial Eta Squared \\
\hline \multirow{2}{*}{ The $1^{\text {st }}$ acquisition } & Practice session & 5 & 568.390 & 21.822 & 0.000 & 0.347 \\
& group & 3 & 560.985 & 3.302 & 0.090 & 0.195 \\
\multirow{3}{*}{ The $2^{\text {nd }}$ acquisition } & Group*practice & 15 & 39.246 & 1.507 & 0.227 & 0.099 \\
& Practice session & 2 & 39.696 & 0.515 & 0.600 & 0.017 \\
& group & 2 & 2513.858 & 13.311 & 0.000 & 0.470 \\
& Group*practice & 4 & 23.507 & 0.305 & 0.873 & 0.020 \\
\hline
\end{tabular}

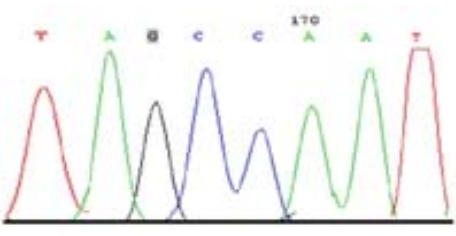

valival

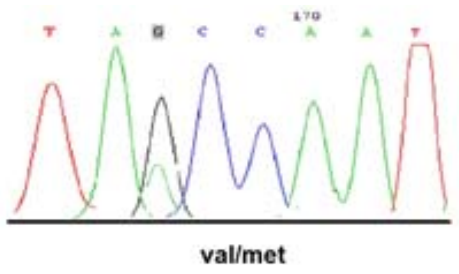

val/met

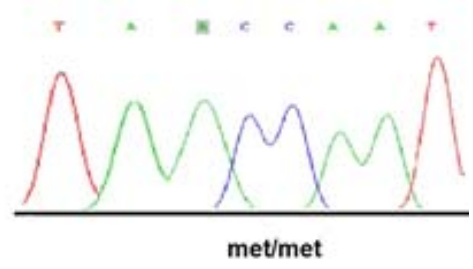

Fig. 1. Sequencing of BDNF gene

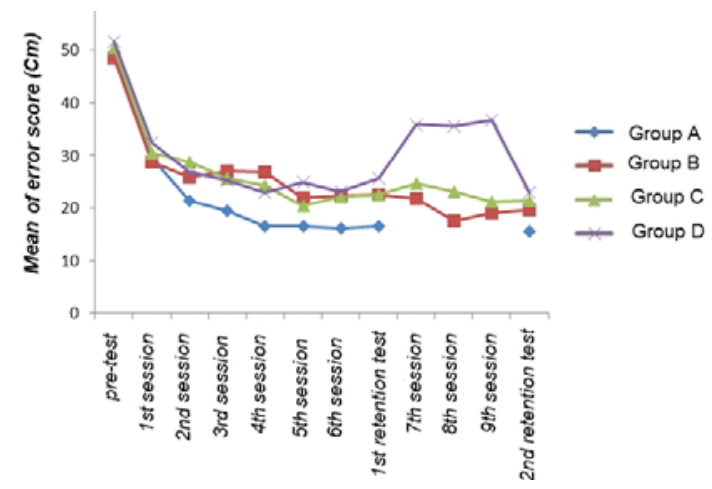

Fig. 2. State of groups in the various stage of training

possibility of moderating this effect by using the most important environmental factors affecting motor learning. Despite the fact that the first retention test showed both groups of the metcarriers were weaker than both groups of people without the polymorphism, only group " $D$ " was significantly weaker than group "A". Therefore, it could be claimed that learning of the motor skill is affected by val66met polymorphism. However, the second retention test showed no significant differences between groups. Hence, it seems that manipulating practice conditions can moderate the influence of val66met polymorphism.

The result of the current study is not consistent with the study of McHughen et al., (2011). They showed that after one day of practice of marble navigation task the met-carriers had a weaker performance than people without the polymorphism, but when the participants continued to practice for 5 days, the difference disappeared. To run the task the participants were seated in front of a board containing nine shallow ( 1 mm deep) wells, arranged in a $3 \times 3$ grid and used the index finger to move the glass marble to successive target wells identified on a computer screen $^{11}$. In the present study by a similar design to McHughen et al.,, the process of learning of group "B" compared with the process of learning of group " $\mathrm{C}$ ". As shown in Figure 2 both groups continued practice with equal error score in the first phase of practice, and there was no difference between them in the first retention test. During the additional practice sessions, while the error score of group "B" was decreased, difference between the groups is not significant neither at practice sessions nor at the second retention test. It is possible that the main reason of difference between the results of the McHughen et al., study and the present study is the difference in the type of the task that was performed. The more a task has components, the more complex it becomes. However, the difficulty of a task is dependent on the relationship between its components ${ }^{21}$. As a result, the task in the present study is very complex and difficult while the task in McHughen et al., is very simple. Moreover, the task in McHughen et $a l$. , is dependent on the motion of one finger and 
is greatly related to the speed of information processing. It is possible that more complex and difficult tasks are affected by other important variables, which are unknown, and overcome the influence of the polymorphism.

From the second practice session, group "A" started to make difference with groups " $C$ " and "D". At the next sessions this difference was remarkable especially with group " $D$ " in a way that it was significant in the first retention test. Result of this phase of the study can be consistent or inconsistent with the previous studies depending on which group of the met-carriers to be considered. In short, some factors such as the type of task, which has already been discussed in detail, and lack of sexuality control in most previous studies may be reasons for difference between the result of the present study and the results of the previous studies. It has been shown that estrogen, a female sex hormone, stimulates expression of BDNF. ${ }^{[16]}$ This issue confuses conclusion about the prevention of BDNF's expression by val66met polymorphism.

Regardless of previous studies results, the present study tried to determine the influence of environmental variables on moderating genetic factors by given protocol. After first retention test while group " $\mathrm{C}$ " continued practicing, group " $\mathrm{A}$ " remained without practice. It is expected that as a result of additional practice group " $\mathrm{C}$ " continues to make progress and compensates the difference with group "A", or even surpass. However, as shown in Figure 2, group "A" benefited from nopractice period, and difference between the groups was remained. Hence, it may be concluded that benefit of no-practice period for people without val66met polymorphism is not lower than the benefit gained by met-carriers who continue to practice in the primer order. The issue could be explained by previous studies in which it is indicated that the lack of practice within hours or days stabilizes memory traces created in the acquisition period and makes neural substrates resistant. ${ }^{[22,23]}$ This concept, called memory consolidation, can lead to increased resistance to interference or even an improvement in performance after a no-practice period. ${ }^{[23]}$ In fact, motor skills are completed by two different processes. Skill can develop during practice (acquisition) sessions called on-line learning, and also develop off-line -between practice sessions or during a no-practice period ${ }^{24}$. It has also been shown that even though BDNF contributes to both acquisition and memory consolidation in a variety of learning tasks ${ }^{8}$, the neural principles operating during consolidation may differ from those during practice $^{24}$. So, the claim is thought-provoking that the benefit of off-line learning for people without the polymorphism is not lower than the benefit of continuing a short term and changeless practice.

However, when practice was associated with a change in the method of providing KR, the results changed. In other words, the results of the second retention test showed that the difference in the first retention test between group " $\mathrm{D}$ " and group "A" has been largely compensated. Due to the results of group "C", it is hard to claim that compensation of the weakness by group " $\mathrm{D}$ " is a result of additional practice ratio to group " $\mathrm{A}$ ". It could be claimed, however, that it is a result of self-controlled KR advantages. As shown in Figure 2, blocking of KR by killing lights degraded the performance of subjects. This drop is not surprising because the given method prevents informative role of feedback and also makes an unusual circumstance. However, the advantage of blocking KR appeared in the second retention test. The method used to provide feedback is very useful because it provides self-controlled KR and prevents immediate KR and $100 \% \mathrm{KR}$. When KR is provided by self-control, it has been proven that the learner participates more actively to determine the characteristics of practice ${ }^{21}$ and also adapts the necessity of additional feedback with his immediate executive requirements ${ }^{1}$. Self-controlled learners are stable, motivated, and independent to reach the goal as well as metacognitively engaged in processes of learning ${ }^{25}, 26$.

Moreover, immediate KR provides too much information for learners causing them to rely on it too heavily; therefore, the subject is not forced to learn the information-processing activities critical for performance. Delayed KR provides much less guidance, and presumably forces the subject to learn the task in a somewhat different way, perhaps by promoting the learner to gather information through alternative feedback sources. ${ }^{[1]}$ In fact, in the delayed time between end of a trial and knowledge of its result the learner actively engages in some processes including 
perception of internal feedback, subjective estimation of error, and problem solving process. As a result, delayed KR can promote error detection capability ${ }^{2}$. It has been demonstrated that as the learner progresses in the skill, the tendency to use vision information to identify the trajectory of the object diminishes. Consequently, the learner detects movement error by detection of a slight deviation in the desired angle of release or in the energy imparted to the ball ${ }^{27}$.

On the other hand, providing of KR on every trial (relative frequency of 100\%) makes an overload on the attention capacity of the learner in a way that, after a few trials, the available information will be more than the ability of learner to process it. ${ }^{[21]}$ Subjects in conditions of lower relative frequency, however, do not have such a strong performance enhancement from KR and are therefore forced to engage in other processes during the acquisition phase. These processes result in the subjects' learning something fundamentally different, such as the capability to detect one's own errors or to be consistent. Perhaps reducing the relative frequency also encourages one to make between-task comparisons, which might facilitate the abstraction of common movement attributes ${ }^{1}$. In addition, when each trial is followed by information about errors, there is a tendency for the subject to make a change for the next trial based on that error. The problem is that motor performance is variable, and a change meant to correct a very small error might actually make the error longer on the next trial. The idea is that KR induces movement variability, not all of which is adaptive in producing improved learning ${ }^{28}$, 29.

In conclusion, the study indicates val66met polymorphism can affect learning of complex motor skills. However, it seems that this effect may be moderated by environmental variables and in certain conditions. In other words, additional practice simply cannot moderate the effect, while the effect may be moderated by changing the condition of practice for people affected by the polymorphism in the way that engages them to cognitive processes. It is recommended to speak with caution about the results and in order to confirm the results more studies should be done in the future.

\section{ACKNOWLEDGMENTS}

Manuscript's Source of support is Ferdowsi University of Mashhad, Iran. Certification code of project from which the paper is derived is: 3/37539.

\section{REFERENCES}

1. Schmidt RA, Lee TD. Motor Control and Learning. Human Kinetics. $5^{\text {th }}$ ed. 2011.

2. Swinnen SP, Schmidt, RA, Nicholson, DE, Shapiro DC. Information feedback for skill acquisition: Instantaneous knowledge of results degrades learning. Journal of Experimental Psychology: Learning, Memory, and Cognition. 1990; 16(4): 706-716.

3. Griffin EW, Bechara RG, Birch AM, Kelly A. M. Exercise enhances hippocampal-dependent learning in the rat: Evidence for a BDNF-related mechanism. Hippocampus 2009; 19(10): 973980.

4. Egan MF, Kojima M, Callicott JH, Goldberg TE, Kolachana BS, Bertolino A, et al., The BDNF val66met polymorphism affects activitydependent secretion of BDNF and human memory and hippocampal function. Cell. 2003; 112(2): 257-69.

5. Joundi RA, Lopez-Alonso V, Lago A, Brittain JS, Fernandez-Del-Olmo M, Gomez-Garre P, et al., The effect of BDNF val66met polymorphism on visuomotor adaptation. Exp Brain Res. 2012; 223(1): 43-50.

6. Freundlieb N, Philipp S, Schneider SA, Bruggemann N, Klein C, Gerloff C, et al., No association of the BDNF val66met polymorphism with implicit associative vocabulary and motor learning. PLoS One. 2012; 7(11): e48327.

7. Pezawas L, Verchinski BA, Mattay VS, Callicott JH, Kolachana BS, Straub RE, et al., The brainderived neurotrophic factor val66met polymorphism and variation in human cortical morphology. J Neurosci. 2004; 24(45): 1009910102.

8. Bramham CR, Messaoudi E. BDNF function in adult synaptic plasticity: the synaptic consolidation hypothesis. Prog Neurobiol. 2005; 76(2):99-125.

9. McHughen SA, Rodriguez PF, Kleim JA, Kleim ED, Marchal Crespo L, Procaccio V, et al., BDNF val66met polymorphism influences motor system function in the human brain. Cereb Cortex. 2010; 20(5): 1254-62. 
10. Hariri AR, Goldberg TE, Mattay VS, Kolachana BS, Callicott JH, Egan MF, et al., Brain-derived neurotrophic factor val66met polymorphism affects human memory-related hippocampal activity and predicts memory performance. $J$ Neurosci. 2003; 23(17): 6690-4.

11. McHughen SA, Pearson-Fuhrhop K, Ngo VK, Cramer SC. Intense training overcomes effects of the Val66Met BDNF polymorphism on short-term plasticity. Exp Brain Res. 2011; 213(4): 415-22.

12. Shimizu E, Hashimoto K, Iyo M. Ethnic difference of the BDNF 196G/A (val66met) polymorphism frequencies: the possibility to explain ethnic mental traits. Am J Med Genet B Neuropsychiatr Genet. 2004; 126B(1): 122-3.

13. Bath KG, Lee FS. Variant BDNF (Val66Met) impact on brain structure and function. Cogn Affect Behav Neurosci. 2006; 6(1): 79-85.

14. Pivac N, Kim B, Nedic G, Joo YH, KozaricKovacic D, Hong JP, et al., Ethnic differences in brain-derived neurotrophic factor Val66Met polymorphism in Croatian and Korean healthy participants. Croat Med J. 2009; 50(1): 43-8.

15. Carbone DL, Handa RJ. Sex and stress hormone influences on the expression and activity of brainderived neurotrophic factor. Neuroscience. 2013; 239: 295-303.

16. Zhou J, Zhang H, Cohen RS, Pandey SC. Effects of estrogen treatment on expression of brainderived neurotrophic factor and cAMP response element-binding protein expression and phosphorylation in rat amygdaloid and hippocampal structures. Neuroendocrinology. 2005; 81(5): 294-310.

17. Gajewski PD, Hengstler JG, Golka K, Falkenstein M, Beste C. The Metallele of the BDNF Val66met polymorphism enhances task switching in elderly. Neurobiol Aging. 2011; 32: 2327 e7-19.

18. Beste C, Baune BT, Domschke K, Falkenstein M, Konrad C. Paradoxical association of the brain-derived-neurotrophic-factor val66met genotype with response inhibition.
Neuroscience. 2010; 166(1): 178-84.

19. Tonacci A, Borghini A, Mercuri A, Pioggia G, Andreassi MG. Brain-derived neurotrophic factor (Val66Met) polymorphism and olfactory ability in young adults. $J$ Biomed Sci. 2013; 20: 57.

20. Horn RR, Williams AM, Hayes SJ, Hodges NJ, Scott MA. Demonstration as a rate enhancer to changes in coordination during early skill acquisition. J Sports Sci. 2007; 25(5): 599-614.

21. Magill RA. Motor learning and control: Concepts and applications. McGraw-Hill Education. $7^{\text {th }}$ ed. 2003.

22. Krakauer JW, Shadmehr R. Consolidation of motor memory. Trends Neurosci. 2006; 29(1): 58-64.

23. Janacsek K, Nemeth D. Predicting the future: from implicit learning to consolidation. Int $J$ Psychophysiol. 2012; 83(2): 213-21.

24. Cohen DA, Pascual-Leone A, Press DZ, Robertson EM. Off-line learning of motor skill memory: a double dissociation of goal and movement. Proc Natl Acad Sci U S A. 2005; 102(50): 18237-41.

25. Zimmerman BJ. Self-regulated learning and academic achievement. An overview. Educational Psychologist. 1990; 25(1): 3-17.

26. Chiricowsky S, Wolf G. Self-control feedback: Does it enhance learning because performances get feedback when they need it? Research Quarterly for Exercise \& Sport. 2002; 73(4): 408-415.

27. Beek P, Lewbel A. The science of juggling. Scientific American. 1995; 273(5): 92-97.

28. Schmidt RA. Frequent augmented feedback can degrade learning: Evidence and interpretations. In: Requn J, Stelmatch GE, editors: Tutorials in motor neuroscience. Dordrecht: Kluwer; 1991. p. 59-75.

29. Schmidt RA, Bjork RA. New conceptualisations of practice; common principles in three paradigms suggest new concepts for training. Psychol Sci. 1992; 3(4): 207-217. 